\title{
Success Rate and Perinatal Outcomes of Selective Reduction by Radiofrequency Ablation in Monochorionic Multiple Pregnancies with a Hydropic Fetus; A Prospective Interventional Single-Arm Study
}

Fatemeh Rahimi Sharbaf, MDi; Mahboobeh Shirazi, MD²; Mansooreh Haghiri, MD²; Mamak Shariat, $\mathrm{PhD}^{2}$; Shirin Niroomanesh, MD'; Marjan Ghaemi, MD; Sakineh Mirzaei Khalilabadi, MD $^{4 *}$

${ }^{1}$ Yas Hospital, Tehran University of Medical Sciences, Tehran, Iran

${ }^{2}$ Maternal, Fetal and Neonatal Research Center, Tehran University of Medical Sciences, Tehran, Iran

${ }^{3}$ Vali-e-Asr Reproductive Health Research Center, Tehran University of Medical Sciences, Tehran, Iran

${ }^{4}$ Department of Obstetrics and Gynecology, School of Medicine, Rafsanjan University of Medical Sciences, Rafsanjan, Iran

\begin{abstract}
Background: Selective reduction of the fetus is one of the therapeutic methods in complicated monochorionic twins. The present study aimed to investigate the success rate and perinatal outcomes of selective reduction using radiofrequency ablation (RFA) in monochorionic multiple pregnancies with a hydropic fetus.

Methods: This prospective interventional single-arm study was conducted in a referral hospital from 2017 to 2019 . Totally, 27 complicated monochorionic twin and triplet pregnancies underwent RFA to occlude the umbilical vein of the hydropic fetus. The participants were followed until 2 months post-delivery. Procedure success rate, survival rate, gestational age at delivery, and neonatal outcome were evaluated.

Results: The umbilical cord in 26 (96.2\%) cases was successfully occluded by RFA with no maternal complications. Live birth was within $75 \%$. The mean \pm SD age at delivery was $34.4 \pm 3.83$ weeks. The gestational age at delivery in TTTS (twin-to-twin transfusion syndrome)-induced hydrops was significantly lower than the non-TTTS hydrops group $(P=0.003)$. Four cases experienced preterm premature rupture of the membranes (PPROM) before 34 weeks. Of all viable neonates, 8 cases were admitted to the neonatal intensive care unit (NICU). In 2 months follow-up post-delivery, all neonates had normal findings without any adverse outcomes. Conclusion: Selective reduction by RFA in complicated monochorionic pregnancies with a hydropic fetus may be a safe method with a high success rate and $100 \%$ neonatal survival. This method may be optimal for feticide in non-TTTS hydropic twins. Keywords: Radiofrequency ablation, Hydropic fetus, Monochorionic multiple pregnancy, Successful rate, Perinatal Outcome Cite this article as: Rahimi Sharbaf F, Shirazi M, Haghiri M, Shariat M, Niroomanesh S, Ghaemi M, et al. Success rate and perinatal outcomes of selective reduction by radiofrequency ablation in monochorionic multiple pregnancies with a hydropic fetus; a prospective interventional single-arm study. Arch Iran Med. 2022;25(1):6-11. doi: 10.34172/aim.2022.02
\end{abstract}

Received: November 20, 2020, Accepted: June 21, 2021, ePublished: January 1, 2022

\section{Introduction}

Twin pregnancy occurs in $3 \%$ of all births. ${ }^{1,2}$ During the recent decades, the prevalence of twin pregnancy has been dramatically increased according to high maternal age, and widespread use of ART (assisted reproductive technique) as the main reasons. ${ }^{2-4}$ Perinatal mortality and morbidity rates related to twin pregnancies are 3-7 times higher than singleton pregnancies and are more common in monochorionic twin pregnancies. ${ }^{2}$

All monochorionic placentas have some degrees of anastomoses. ${ }^{5}$ Shunting may occur between the two fetuses with blood pressure or blood flow gradient. ${ }^{6}$ This chronic situation of fetal-to-fetal blood transfusion can lead to severe complications unique to monochorionic twin pregnancy such as Twin-to-Twin Transfusion Syndrome (TTTS), selective fetal growth restriction (sFGR), twin reversed arterial perfusion sequence (TRAP), and twin anemia-polycythemia sequence (TAPS). ${ }^{3}$
Furthermore, the congenital anomaly rate in monochorionic twins is 633.6 per $10000 .{ }^{7}$ In cases with the demise of one fetus, the remaining fetus encounters some complications due to blood transfusions through placental anastomosis which results in brain hypoperfusion, brain damage (20\% to $30 \%$ ) and the death of the co-twin (10\%). ${ }^{8}$

Selective reduction of the fetus is one of the therapeutic methods in complicated monochorionic twins. ${ }^{9}$ Typically, in the absence of intervention, both fetuses may die. However, due to the shared blood circulation, any injection of feticide agents into one fetus imposes some adverse effects on the other fetus. ${ }^{10}$ Therefore, different techniques such as the closure of the umbilical vein or cord via radiofrequency ablation (RFA), fetoscopic ligation or laser coagulation and monopolar or bipolar energy are available for selective reduction.

The mechanism of RFA is alternating current with very high frequencies through the needle. Tissue ions become 
agitated as they attempt to align with the electrical field. The generated heat causes tissue coagulation and necrosis. ${ }^{5}$

RFA has some advantages including safety and efficacy for different indications. ${ }^{11}$ Nevertheless, all selective feticide methods have some risks. A systematic review study showed that the highest survival rate of the remaining monochorionic fetus pertained to RFA (86\%) followed by cord closure with bipolar cautery $(82 \%)$, cord coagulation by laser (72\%), and cord closure (70\%), in decreasing order. ${ }^{12}$

In the case of complicated monochorionic twin pregnancies with one hydropic fetus, selective feticide using laser or bipolar cautery may be more difficult due to edema of the umbilical cord that may reduce the success rate. Moreover, use of RFA seems difficult due to abdominal ascites and may affect the success rate and subsequent perinatal outcomes.

A small number of studies have evaluated selective reduction using RFA for the treatment of monochorionic twin pregnancies complicated by a hydropic fetus. This prospective study was designed to determine the success rate and fetal and neonatal outcomes of selective reduction by RFA.

\section{Materials and Methods}

After selecting the patients, RFA was explained. Prior to any procedure, all steps and related risks including miscarriage, preterm labor, probability of any morbidity, and mortality in the co-twin were discussed for eligible participants. Written informed consent was taken from all participants. This prospective interventional single-arm study was conducted at Yas Hospital affiliated to Tehran University of Medical Sciences (Tehran, Iran) from January 2017 to August 2019.

Of 271 cases who were candidates for selective reductions by RFA due to complications related to monochorionic twins and triplets, 27 cases $(9.96 \%)$ with a hydropic fetus entered the study.

The inclusion criteria were monochorionic multiple pregnancies with at least one monochorionic twin, gestational age of 16 to 26 weeks (based on first trimester ultrasound), presence of a hydropic fetus and willingness to participate in the study. The exclusion criteria were a medical prohibition to perform RFA. Indeed, firsttrimester combine test or non-invasive prenatal testing was requested for all patients. In the case of positive screening or non-TTTS hydrops, amniocentesis for both twins was performed. Mothers with abnormal amniocentesis were also excluded from the study.

A questionnaire containing demographic and current obstetrical data was completed for each patient who accepted the RFA method. A contact number was obtained from subjects for follow-up.

TTTS staging was performed according to Quintero et $\mathrm{al}^{13}$ : stage I, bladder in the donor twin (BDT) still visible; stage II, BDT no longer visible, no critically abnormal Doppler studies (CADs); stage III, CADs; stage IV, hydrops; stage V, demise of one or both twins.

The day before the procedure, ultrasound examination was performed by an expert perinatologist to confirm RFA indication, biometric assessment including estimation of fetal weight, amniotic fluid volume and Doppler study for each fetus. In the cases of severe polyhydramnios and severe uterine distention, amnioreduction was performed one day before RFA until the largest amniotic fluid pocket reached less than $8 \mathrm{~cm} .{ }^{14}$

\section{Technique}

About 30 minutes before RFA, $50 \mathrm{mg}$ rectal indomethacin suppository, $1 \mathrm{~g}$ oral amoxicillin and $50 \mathrm{mg}$ intramuscular promethazine were prescribed. Fetal heart was checked before the procedure. Doppler ultrasound and reevaluation of RFA indication were also done. ${ }^{14}$

RFA was performed using a radiofrequency generator (RF um 2004, manufactured by RF medical Co. of South Korea). After local disinfection and injection of $2 \%$ lidocaine solution, under continuous ultrasound and color Doppler guidance, a simple 17-gauge needle (2 $\mathrm{cm}$ exposed tip) with an internally cooled electrode was inserted into the mother's uterus. ${ }^{14}$ In a hydropic fetus with abdominal skin diameter $\geq 1.5 \mathrm{~mm}$, the needle was inserted through the skin and entered the umbilical cord at the site of entry into the abdomen. In cases with the absence of skin edema and low ascites, the needle entered the umbilical vein into the abdomen. If ascites were notable, the needle was entered into the fetal liver and the umbilical vein was coagulated (Figure 1).

Before radiofrequency energy application, the needle tip position was determined by color mapping. ${ }^{5}$ The umbilical vein of the fetus was cauterized at a $100 \mathrm{~W}$ current for a maximum of 2 minutes (with needle entrance to the liver, the energy was given for a shorter time due to the fragility of liver tissue).

At the end of the procedure, cease of blood flow was confirmed by color Doppler and fetal heart rate was

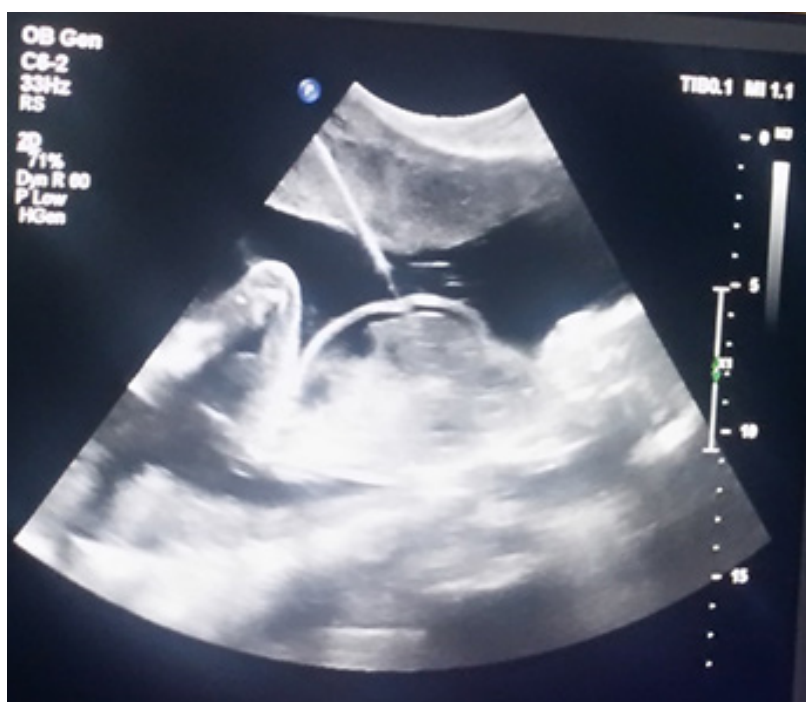

Figure 1. RFA Procedure by Passing a Needle Via the Fetal Abdomen of a Hydropic Fetus. 
gradually decreased until asystole. If blood flow was not interrupted, the procedure was repeated and the fetal heart rate was monitored by ultrasonography until asystole. ${ }^{14}$

The day after the procedure, all patients underwent ultrasound examination to evaluate the success of the procedure and viability of the co-twin as well as measurement of middle cerebral arterial peak systolic velocity (MCA-PSV) to detect fetal anemia. MCA PSV higher than 1.5 multiples of median (MOM) was considered anemic ${ }^{15}$ and for sFGR definition, the Delphi criteria were applied. ${ }^{16}$

Patients were followed weekly. After one month, the perinatal routine care by obstetrician was recommended to detect any complications such as chorioamnionitis, PPROM, spontaneous abortion, intrauterine fetal death (IUFD), and preterm delivery. Finally, delivery time, gestational age at delivery, type of delivery, infant characteristics, and NICU admission were recorded. Indeed, we compared the perinatal outcome of the TTTS hydropic and sFGR cases with normal. All neonates were followed 2 months post-delivery to detect any potential complications.

\section{Data Analysis}

The data were analyzed using SPSS software version 18.0. Quantitative data were reported as mean \pm standard deviation and qualitative data were reported as number (\%). Independent $t$ test and Fisher's exact test were used for data analysis. It should be noted that before performing an independent $t$ test, the normality of distributionrelated variables was evaluated by Kolmogorov-Smirnov nonparametric test. The significance level was considered at 0.05 .

\section{Results}

Twenty-seven monochorionic multiple pregnancies with a hydropic fetus were recruited in this study. The demographic and obstetrical characteristics of mothers are summarized in Table 1.

Sixteen (59.3\%) cases had TTTS Stage IV and 11 cases (40.7\%) had non-TTTS hydrops due to fetal heart anomaly, cystic hygroma, Turner syndrome, and unknown causes. In 10 cases, amnioreduction was performed due to severe polyhydramnios ( 9 cases because of TTTS Stage IV and one case due to fetal anomaly).

In 26 cases (96.3\%), the umbilical vein was successfully occluded by RFA and the fetuses became asystolic at the end of the procedure.

There were no maternal complications associated with the procedure. In one case, intra-amniotic hemorrhage occurred from the site of the needle insertion into the placenta, but in the postoperative examination, the mother and fetus were not anemic.

Ultrasound evaluation one day after the procedure showed four cases with fetal anemia. In one case of failed RFA, both fetuses died after the intervention.

Abortion occurred in four cases at a gestational age
Table 1. Demographic and Pregnancy Characteristics of the Participants

\begin{tabular}{|c|c|c|}
\hline Characteristics & \multicolumn{2}{|c|}{ Values } \\
\hline Age $($ Mean $\pm S D$; years $)$ & \multicolumn{2}{|c|}{$31.07 \pm 6.81$} \\
\hline $\mathrm{BMI}\left(\right.$ Mean $\left.\pm \mathrm{SD} ; \mathrm{kg} / \mathrm{m}^{2}\right)$ & \multicolumn{2}{|c|}{$29.51 \pm 4.56$} \\
\hline \multirow{2}{*}{ Parity, n (\%) } & Nullipara & $12(44.4 \%)$ \\
\hline & Multipara & $15(55.6 \%)$ \\
\hline \multirow{3}{*}{ Conception, n (\%) } & Spontaneous & $22(81.48 \%)$ \\
\hline & Induction Ovulation & $2(7.40 \%)$ \\
\hline & IVF & $3(11.11 \%)$ \\
\hline \multirow{3}{*}{ Chorionicity, n (\%) } & MCDA & $25(92.6 \%)$ \\
\hline & MCMA & $1(3.7 \%)$ \\
\hline & MCTA & $1(3.7 \%)$ \\
\hline \multirow{2}{*}{ TTTS (n (\%) } & Yes & $16(59.3 \%)$ \\
\hline & No & $11(40.7 \%)$ \\
\hline $\begin{array}{l}\text { GA at procedure } \\
(\text { Mean } \pm S D ; \text { weeks })\end{array}$ & \multicolumn{2}{|c|}{$21.79 \pm 2.87\left(16-26^{+4}\right)$} \\
\hline
\end{tabular}

BMI, body mass index; IVF, In vitro Fertilization; MCDA, Monochorionic diamniotic; MCMA, monochorionic-monoamniotic; MCTA, monochorionictriamniotic; TTTS, Twin-to-twin transfusion syndrome; GA, gestational age.

under 24 weeks; one of the abortions was due to failure of the procedure and the other three because of fetal anemia. In two cases, abortion was requested by parents. In another case, spontaneous abortion happened 5 days after the procedure at $22^{+4}$ weeks of gestational age.

Out of the 26 successful cases, IUFD was observed in four cases (15.3\%). One IUFD was in a monochorionic monoamniotic pregnancy of which selective reduction was performed at $18^{+3}$ weeks due to hydrops and cardiac abnormality of one fetus, but at $26^{+1}$ weeks, IUFD occurred due to cord twisting. The second case was observed in pregnancy with TTTS Stage IV, where RFA was performed on a hydropic fetus at $21^{+4}$ weeks, but IUFD occurred one month later at $25^{+2}$ weeks due to an unknown cause. Another case was a pregnant woman, for whom selective reduction with RFA was performed at $22^{-1}$ weeks due to TTTS Stage IV. The day after the operation, the other fetus was anemic, due to ascites and hydrops; termination of pregnancy did not happen because of the mother's refusal and IUFD occurred at $22^{+4}$ weeks ( 5 days after the procedure). The other IUFD happened in a case after successful RFA procedure at $26^{-1}$ weeks of gestation due to cardiac anomaly and fetal hydrops but she experienced placenta abruption and IUFD at $28^{+4}$ weeks consequent to severe trauma.

Except for abortion and IUFD, the live births in the study were 20 cases $(74 \%)$. One case was delivered before 26 weeks $\left(25^{+3}\right.$ weeks $)$ that could not survive due to prematurity. The mean \pm SD of gestational age at delivery was $34.4 \pm 3.83$ weeks (with the range of $27^{+3}$ to $40^{-1}$ ).

The mean birth weight among 19 viable cases at birth was 2276 grams (range 1100 to 3400 ). Out of 23 cases, 10 cases $(43.5 \%)$ had a normal vaginal delivery (NVD) and 13 cases $(56.5 \%)$ had a cesarean section. The most common causes of cesarean section were previous cesarean section and non-cephalic fetal presentation. 
Four cases experienced PPROM before 34 weeks. PPROM in only one case was before 4 weeks of the procedure. All PPROM cases occurred in the TTTS group but without a significant difference $(P=0.10)$ compared to the non-TTTS hydrops groups.

The mean gestational age at delivery in TTTS-induced hydrops was 32.5 weeks with a significant difference $(P=0.003)$ compared to non-TTTS hydrops with a mean gestational age of 37.2 weeks at delivery. The gestational age at delivery in live births was statistically significant between the two groups with or without amnioreduction (30.3 vs. 36.3 weeks; $P<0.001$ ).

Eight cases had delivery before 34 weeks (early preterm), of which TTTS was observed as a cause of hydrops in seven cases. Delivery in the other 11 (57.9\%) cases happened after 34 weeks, of which TTTS was observed in four cases. Results showed that there was no significant relationship between TTTS and early preterm delivery $(P=0.059)$.

Out of 27 participants, 16 (59.3\%) cases had nonhydropic fetuses with selective intrauterine growth restriction, of which seven cases were TTTS. Therefore, there was not any significant correlation between TTTS and $\operatorname{sFGR}(P=0.10)$.

Eleven cases of viable deliveries had sFGR fetus at the time of the procedure with a mean \pm SD gestational age of $35.3 \pm 3.8$ weeks; compared to normal fetuses $(34 \pm 3.87$ weeks), there was no significant difference $(P=0.40)$. The mean \pm SD birth weights in groups with and without sFGR in delivery were $2345 \pm 751$ and $2181 \pm 558$ g, respectively, with no significant difference $(P=0.60)$.

Eight newborns were admitted to the NICU and all were discharged without any permanent complications. All neonates were followed up for 2 months post-delivery that revealed no complications. Characteristics related to pregnancies and deliveries of 19 cases with viable births are listed in Table 2.

\section{Discussion}

RFA seems to be a safe and low-risk option for both mother and fetus in complicated multiple pregnancies to ablate the abnormal fetus. The procedure success rate in this study was $96 \%$. Note that if this intervention was not performed, the fetal mortality and long-term complications would be higher than $80 \%$. Indeed, no long-term and serious complications were observed in newborns.

This rate is similar to other studies. In studies by Dadhwal et al, ${ }^{8}$ Bebbington et $a l,{ }^{17} \mathrm{Lu}$ et $\mathrm{al}^{18}$ and Kumar et $\mathrm{al},{ }^{5}$ the success rate was near $100 \%$, whereas in a study by Rossi and D'Addario, failure of feticide occurred in 9\% of RFA cases. ${ }^{12}$ Paramasivam et al reported 35 cases using RFA: the technical successful rate was $100 \%$; however, there were only three cases of TTTS Stage IV. ${ }^{19}$ In comparison, in our study, all cases were hydropic and as mentioned, this process is more complex in such cases. Besides, the spontaneous abortion rate was $7 \%$ which was also similar to other studies. ${ }^{5,20}$

The live birth rate was near $75 \%$. In the study by Lu et al, the total survival rate was $81.8 \%$ but there was only

Table 2. Pregnancy and Delivery Specifications among 19 Viable Cases

\begin{tabular}{|c|c|c|c|c|c|c|c|c|}
\hline TTTS & $\begin{array}{l}\text { sFGR at } \\
\text { Procedure }\end{array}$ & Amnioreduction & $\begin{array}{c}\text { GA at } \\
\text { Procedure }(w)\end{array}$ & $\begin{array}{c}\text { GA at Delivery } \\
(\mathbf{w})\end{array}$ & $\begin{array}{l}\text { Type of } \\
\text { Delivery }\end{array}$ & Cause of Delivery & Birth Weight & $\begin{array}{c}\text { NICU } \\
\text { Admission }\end{array}$ \\
\hline- & + & - & 16 & 38 & $\mathrm{C} / \mathrm{S}$ & Repeat & 3100 & - \\
\hline+ & - & + & $23+5$ & $37+4$ & $\mathrm{C} / \mathrm{S}$ & Elective & 2300 & - \\
\hline - & + & - & 20 & $37+2$ & $\mathrm{C} / \mathrm{S}$ & Elective & 3400 & - \\
\hline+ & - & + & $25+1$ & $27+3$ & $\mathrm{C} / \mathrm{S}$ & Elective (PPROM) & 1800 & + \\
\hline- & - & - & $19+5$ & 38 & NVD & Spontaneous & 2600 & - \\
\hline+ & - & - & 20 & 31 & $\mathrm{C} / \mathrm{S}$ & Elective (Twin + PPROM) & $\begin{array}{l}1300 \\
1450\end{array}$ & + \\
\hline+ & + & + & $22+4$ & $35+1$ & $\mathrm{C} / \mathrm{S}$ & Repeat (preterm labor) & 2700 & - \\
\hline+ & - & - & $19+1$ & $37+2$ & $\mathrm{C} / \mathrm{S}$ & Repeat & 2600 & - \\
\hline+ & + & + & $23+2$ & $28+4$ & $\mathrm{C} / \mathrm{S}$ & Repeat (PPROM) & 1310 & + \\
\hline- & - & - & $18+4$ & 37 & NVD & ROM & 3100 & - \\
\hline+ & - & + & $26+3$ & 33 & $\mathrm{C} / \mathrm{S}$ & Repeat (preterm labor) & 1800 & + \\
\hline - & + & - & $22+1$ & $37+6$ & $\mathrm{C} / \mathrm{S}$ & Repeat & 2500 & + \\
\hline+ & - & + & $26+3$ & $31+2$ & NVD & Preterm labor & 1800 & + \\
\hline- & + & - & $19+1$ & $37+3$ & $\mathrm{C} / \mathrm{S}$ & Repeat (preterm labor) & 2100 & - \\
\hline+ & + & - & $18+6$ & $38+6$ & NVD & Spontaneous & 2420 & - \\
\hline+ & + & + & $25+5$ & $28+5$ & NVD & Preterm labor & 1100 & + \\
\hline+ & + & - & $21+1$ & $33+2$ & $\mathrm{C} / \mathrm{S}$ & Repeat (PPROM & 1600 & + \\
\hline- & + & - & $26+4$ & $39+4$ & NVD & Spontaneous & 3090 & - \\
\hline- & + & - & 20 & $34+4$ & $\mathrm{C} / \mathrm{S}$ & Breech (preterm labor) & 2480 & - \\
\hline
\end{tabular}

TTTS, Twin-to-twin transfusion syndrome; GA, gestational age; sFGR, selective fetal growth restriction; NICU, neonatal intensive care unit; CS, cesarean section; PPROM, preterm premature rupture of membrane; NVD, normal vaginal delivery 
one case of TTTS Stage IV. ${ }^{21}$ In other studies with higher success rates, the TTTS Stage IV cases were reported scarcely ${ }^{18,22}$ and it seems the survival rate decreased to $60 \%$ in TTTS cases. ${ }^{12}$ In 2014, Bebbington showed that of all RFA indications, the survival rate for TTTS was lower than other indications. ${ }^{23}$

Fetal anemia after RFA was $15 \%$ in our study that led to fetal demise. Wang et al examined MSA PSV 24 hours after RFA and found no case with fetal anemia. ${ }^{22}$ Another study reported abnormal MCA- PSV in nearly $10 \%$ of cases. ${ }^{24}$ This difference may be related to the presence of hydropic fetuses and the difficulty of performing the procedure in this group. Therefore, the blood circulation of the hydropic twin was not completely occluded that caused anemia in the normal fetus.

The rate of IUFD was $18.5 \%$ in this report that is slightly lower compared with other similar cases in the study by Bebbington et al. ${ }^{17}$ In a systematic review, the fetal demise rate was reported at $14.7 \% .^{9}$ In a study by Rossi and D'Addario, the rate of IUFD with the RFA method was lower than the other feticide methods. ${ }^{12}$

In our study, the mean gestational age at delivery was $34.47 \pm 3.83$ weeks and the average weight of newborns was 2276 grams. This is similar to other studies., ${ }^{5,95,26}$ Among the available studies, only the study by Bebbington et al had a large number of TTTS Stage IV and gestational age at delivery was 33 weeks. ${ }^{23}$ The reason for the higher preterm delivery rate in the present study may be probably due to severe underlying pathological factors (presence of more than half of hydropic fetuses in TTTS stage IV) and the difficulty in performing the procedure. Regarding the birth weight of newborns, the results of our study were compatible with those of Lu et $\mathrm{al}^{21}$ and Roman et $\mathrm{al}^{18}$ where the birth weights were 2204 and $2350 \mathrm{~g}$, respectively.

The lowest and highest rates of PPROM were reported in the studies by Roman et al (5\%) and Peng et al (33.3\%), respectively. ${ }^{18,24}$ Our result is similar to the other 12 studies that were reviewed by Rossi and D'Addario. ${ }^{12}$ In the present study, all PPROM cases occurred in patients with TTTS but the relationship between TTTS and PPROM was not statistically significant. This may be due to the small number of cases. We could not find any investigations to have assessed this relationship.

It was shown that gestational age at delivery was significantly higher in the non-TTTS hydrops group compared to the TTTS-induced hydrops group. The underlying pathology of TTTS and uterine overdistention may be the cause. In such cases, delivery can occur at a lower gestational age.

On the other hand, in our study, 59.3\% of pregnancies had an sFGR fetus at the time of the procedure, but the relationship between TTTS and sFGR was not statistically significant. On the other hand, in the studies by Van Winden et al and Groene et al, 65\% and 59.2\% of TTTS patients had sFGR fetuses, respectively ${ }^{27,28}$ and the correlation between TTTS and sFGR was statistically significant. In addition, in our study, the relationships between sFGR and gestational age at delivery or birth weight were not significant. These findings may be due to lack of a significant relationship between TTTS and sFGR, as most of the hydrops cases in our study were TTTS.

In the present study, gestational age at delivery in the group that underwent amnioreduction was significantly lower in comparison with the group without amnioreduction. This may also be due to the severity of the disorder in the amnioreduction group.

There were no neonatal deaths in our study, confirming other studies that have reported $100 \%$ neonatal survival. ${ }^{20-22,25,29}$ However, in a study by Roman et al, the rate of neonatal survival was $91.3 \% .^{18}$ It was also shown that infantile mortality was lower with RFA compared to the other methods. ${ }^{9}$

In conclusion, selective reduction by RFA in complicated monochorionic pregnancies with a hydropic fetus may be a safe method with a high success rate and $100 \%$ neonatal survival. This method may be optimal for feticide in nonTTTS hydropic twins either.

\section{Recommendations}

Although the results of this study are acceptable due to the difficulty of intervention, it is suggested to perform a comparative study between this method and Laser coagulation in hydropic fetuses in terms of surgical success and neonatal survival.

\section{Authors' Contribution}

FRS and MS and SS designed the study. Patients were treated by MH, SMk. MS did the statistical analysis. SM and MG wrote the article.

\section{Conflict of Interest Disclosures}

The authors have no conflicts of interest.

\section{Ethical Statement}

This study was approved by the Ethics Committee of Tehran University of Medical Sciences (IR. TUMS.MEDICINE.REC.1498.558).

\section{References}

1. Cunningham F, Leveno K, Bloom S, Dashe J, Hoffman B, Casey B, et al. Williams Obstetrics. McGraw Hill Education; 2018.

2. Resnik R, Lockwood C, Moore T, Greene M, Copel J, Silver R. Creasy and Resnik's Maternal-Fetal Medicine: Principles and Practice. 8th ed. Elsevier; 2018.

3. James D, Steer P, Weiner C, Gonik B, Robson S. High-Risk Pregnancy: Management Options. 5th ed. Cambridge University Press; 2017.

4. Rissanen AS, Jernman RM, Gissler M, Nupponen I, Nuutila ME. Maternal complications in twin pregnancies in Finland during 1987-2014: a retrospective study. BMC Pregnancy Childbirth. 2019;19(1):337. doi: 10.1186/s12884-019-2498-x.

5. Kumar S, Paramasivam G, Zhang E, Jones B, Noori M, Prior $\mathrm{T}$, et al. Perinatal- and procedure-related outcomes following radiofrequency ablation in monochorionic pregnancy. Am J Obstet Gynecol. 2014;210(5):454.e1-454.e6. doi: 10.1016/j. ajog.2013.12.009.

6. Remien K, Majmundar SH. Physiology, fetal circulation. In: StatPearls. Treasure Island, FL: StatPearls Publishing; 2021.

7. Glinianaia SV, Rankin J, Wright C. Congenital anomalies in twins: a register-based study. Hum Reprod. 2008;23(6):130611. doi: 10.1093/humrep/den104. 
8. Dadhwal V, Sharma AK, Deka D, Chawla L, Agarwal N. Selective fetal reduction in monochorionic twins: preliminary experience. J Turk Ger Gynecol Assoc. 2019;20(2):79-83. doi: 10.4274/jtgga.galenos.2018.2018.0052.

9. Gaerty K, Greer RM, Kumar S. Systematic review and metaanalysis of perinatal outcomes after radiofrequency ablation and bipolar cord occlusion in monochorionic pregnancies. Am J Obstet Gynecol. 2015;213(5):637-43. doi: 10.1016/j.ajog.2015.04.035.

10. Pasquini L, Pontello V, Kumar S. Intracardiac injection of potassium chloride as method for feticide: experience from a single UK tertiary centre. BJOG. 2008;115(4):528-31. doi: 10.1111/j.1471-0528.2007.01639.x.

11. Rahimi-Sharbaf F, Ghaemi M, Nassr AA, Shamshirsaz AA, Shirazi M. Radiofrequency ablation for selective fetal reduction in complicated monochorionic twins; comparing the outcomes according to the indications. BMC Pregnancy Childbirth. 2021;21(1):189. doi: 10.1186/s12884-021-036561.

12. Rossi AC, D'Addario V. Umbilical cord occlusion for selective feticide in complicated monochorionic twins: a systematic review of literature. Am J Obstet Gynecol. 2009;200(2):123-9. doi: 10.1016/j.ajog.2008.08.039.

13. Quintero RA, Morales WJ, Allen MH, Bornick PW, Johnson PK, Kruger M. Staging of twin-twin transfusion syndrome. J Perinatol. 1999;19(8 Pt 1):550-5. doi: 10.1038/sj.jp.7200292.

14. Rahimi-Sharbaf F, Shariat M, Niromanesh S, Shirazi M, Soleimani Z. Foetal heart rate pattern after complete cord occlusion by radiofrequency ablation for selective reduction in the complicated monochorionic twin pregnancies. Adv Hum Biol. 2019:9(1):84-8. doi: 10.4103/aihb.aihb_29 18.

15. Andrei C, Vladareanu R. The value of reference ranges for middle cerebral artery peak systolic velocity in the management of rhesus alloimmunized pregnancies. Maedica (Bucur). 2012;7(1):14-9.

16. Khalil A, Beune I, Hecher K, Wynia K, Ganzevoort W, Reed K, et al. Consensus definition and essential reporting parameters of selective fetal growth restriction in twin pregnancy: a Delphi procedure. Ultrasound Obstet Gynecol. 2019;53(1):47-54. doi: 10.1002/uog.19013.

17. Bebbington MW, Danzer E, Moldenhauer J, Khalek N, Johnson MP. Radiofrequency ablation vs bipolar umbilical cord coagulation in the management of complicated monochorionic pregnancies. Ultrasound Obstet Gynecol. 2012;40(3):319-24. doi: 10.1002/uog.11122.

18. Roman A, Papanna R, Johnson A, Hassan SS, Moldenhauer J, Molina S, et al. Selective reduction in complicated monochorionic pregnancies: radiofrequency ablation vs. bipolar cord coagulation. Ultrasound Obstet Gynecol. 2010;36(1):37-41. doi: 10.1002/uog.7567.
19. Paramasivam G, Wimalasundera R, Wiechec $M$, Zhang E, Saeed F, Kumar S. Radiofrequency ablation for selective reduction in complex monochorionic pregnancies. Bjog. 2010;117(10):1294-8. doi: 10.1111/j.14710528.2010.02624.x.

20. Sun L, Zou G, Yang Y, Zhou F, Tao D. Risk factors for fetal death after radiofrequency ablation for complicated monochorionic twin pregnancies. Prenat Diagn. 2018;38(7):499-503. doi: 10.1002/pd.5269.

21. Lu J, Ting YH, Law KM, Lau TK, Leung TY. Radiofrequency ablation for selective reduction in complicated monochorionic multiple pregnancies. Fetal Diagn Ther. 2013;34(4):211-6. doi: 10.1159/000355406.

22. Wang HM, Li HY, Wang XT, Wang YY, Li L, Liang B, et al. Radiofrequency ablation for selective reduction in complex monochorionic multiple pregnancies: a case series. Taiwan J Obstet Gynecol. 2017;56(6):740-4. doi: 10.1016/j. tjog.2017.10.006.

23. Bebbington M. Selective reduction in complex monochorionic gestations. Am J Perinatol. 2014;31 Suppl 1:S51-8. doi: 10.1055/s-0034-1383852.

24. Peng $R$, Xie HN, Lin MF, Yang JB, Zhou $Y$, Chen $H Q$, et al. Clinical outcomes after selective fetal reduction of complicated monochorionic twins with radiofrequency ablation and bipolar cord coagulation. Gynecol Obstet Invest. 2016;81(6):552-8. doi: 10.1159/000445291.

25. van den Bos EM, van Klink JM, Middeldorp JM, Klumper FJ, Oepkes D, Lopriore E. Perinatal outcome after selective feticide in monochorionic twin pregnancies. Ultrasound Obstet Gynecol. 2013;41(6):653-8. doi: 10.1002/uog.12408.

26. Yinon $Y$, Ashwal E, Weisz B, Chayen B, Schiff E, Lipitz S. Selective reduction in complicated monochorionic twins: prediction of obstetric outcome and comparison of techniques. Ultrasound Obstet Gynecol. 2015;46(6):670-7. doi: 10.1002/ uog. 14879.

27. Groene SG, Tollenaar LSA, van Klink JMM, Haak MC, Klumper F, Middeldorp JM, et al. Twin-twin transfusion syndrome with and without selective fetal growth restriction prior to fetoscopic laser surgery: short and long-term outcome. J Clin Med. 2019;8(7). doi: 10.3390/jcm8070969.

28. Van Winden KR, Quintero RA, Kontopoulos EV, Korst LM, Llanes A, Chmait RH. Perinatal survival in cases of twin-twin transfusion syndrome complicated by selective intrauterine growth restriction. J Matern Fetal Neonatal Med. 2015;28(13):1549-53. doi: 10.3109/14767058.2014.960834.

29. Moise KJ Jr, Johnson A, Moise KY, Nickeleit V. Radiofrequency ablation for selective reduction in the complicated monochorionic gestation. Am J Obstet Gynecol. 2008;198(2):198.e1-198.e5. doi: 10.1016/j. ajog.2007.07.043. 